\title{
The workweek is the best time to start a family - A Study of GPT-2 Based Claim Generation
}

\author{
Shai Gretz*, Yonatan Bilu*, Edo Cohen-Karlik and Noam Slonim \\ IBM Research \\ \{avishaig,yonatanb,noams\}@il.ibm.com \\ \{edo.cohen\}@ibm.com
}

\begin{abstract}
Argument generation is a challenging task whose research is timely considering its potential impact on social media and the dissemination of information. Here we suggest a pipeline based on GPT-2 for generating coherent claims, and explore the types of claims that it produces, and their veracity, using an array of manual and automatic assessments. In addition, we explore the interplay between this task and the task of Claim Retrieval, showing how they can complement one another.
\end{abstract}

\section{Introduction}

Argument Mining had traditionally focused on the detection and retrieval of arguments, and the classification of their types and of the relations among them. Recently, there has been growing interest in argument synthesis. Here we suggest a pipeline for addressing this task relying on the GPT-2 language model (Radford et al., 2019), examine how it can be enhanced to provide better arguments, and analyze the types of arguments being produced. Specifically, we are interested in Claim Generation, where the input is a debate topic, phrased as a proposed policy, and the output is a concise assertion with a clear stance on this topic.

We start by fine-tuning GPT- 2 on a collection of topics and associated claims. Since several such datasets are available, we examine which of them tend to yield better claims, and observe that merging all such sources together does not necessarily yield better results. In addition, we explore two ways in which context can be added to the generation process, beyond providing the topic itself: (i) framing the topic with the first sentence from its corresponding Wikipedia page; and (ii) framing the claim by directing it to consider a specific aspect. We find that the former can improve the generated

\footnotetext{
${ }^{*}$ These authors equally contributed to this work.
}

output, but the latter does not - at least in the way it is done here. Following Bilu and Slonim (2016), we also examine a post-generation ranking step that aims to select the correctly generated claims. We find that existing Claim Detection tools can serve as a filter to significantly enhance generation quality.

Our evaluation incorporates automatic measures and manual labeling. Specifically, we introduce an annotation task aiming to assess the plausibility of generated claims, i.e., to what degree is it plausible that a human will make it. We report results on a test set of 96 topics, demonstrating the validity of our approach to topics not seen in training or development. In addition, we manually annotate the generated claims for whether they are factual claims, or opinion based, and further aim to assess whether the former represent true facts.

Finally, we observe that manually labeled datasets used to fine-tune GPT- 2 are not essential, and that relying on the output of a Claim Retrieval ${ }^{1}$ engine for this fine-tuning, may suffice. In addition, we compare the generated claims to an existing large-scale collection of claims for the same topics, and conclude that the generated claims tend to be novel, and hence may augment traditional Argument Mining techniques in automatically providing claims for a given topic.

Henceforth, we denote the initial output of GPT2 for a given prompt as generated text $(G T)$. Thus, our task is to define a process by which as many of the GTs as possible will represent claims that are relevant to the provided prompt.

\footnotetext{
${ }^{1}$ Given a topic of interest, Claim Retrieval is the task of retrieving relevant claims from a corpus; Claim Detection is the task of determining whether a given text is a relevant claim.
} 


\section{Related Work}

In classical Natural Language Generation (NLG) tasks - Machine Translation, Summarization, and Question Answering - the semantic content of the output strongly depends on the input. Argument Generation, alongside Story Generation (Fan et al., 2018), occupies a parallel venue, where the output should satisfy stylistic and rhetorical constraints - yet no well-defined semantic goal - with much room and desire for innovation.

Approaches to argument generation have included traditional NLG architectures (Zukerman et al., 1998; Carenini and Moore, 2006); assembling arguments from given, smaller argumentative units (Walton and Gordon, 2012; Reisert et al., 2015; Wachsmuth et al., 2018; El Baff et al., 2019); welding the topic of the debate to appropriate predicates (Bilu and Slonim, 2016); and using predefined argument templates (Bilu et al., 2019). Of particular interest is the generation of counter arguments, for which solutions include an encoderdecoder architecture (Hidey and McKeown, 2019), which may be augmented by a retrieval system (Hua et al., 2019; Hua and Wang, 2018), or alternatively offering "general purpose" rebuttal based on similarity to predefined claims (Orbach et al., 2019).

Concurrent with our work, and most similar, is Schiller et al. (2020), who frame the AspectControlled Argument Generation problem as follows - given a topic, a stance and an aspect, generate an argument with the given stance towards the topic, which discusses the given aspect. They finetune CTRL (Keskar et al., 2019) over claims from 8 controversial topics, and mostly use automatic measures to assess claim generation over the same 8 topics. By contrast, here we are interested in a less restricted setting and explore the properties of the generated claims. Specifically, we fine-tune GPT-2 on claims coming from diverse sets of 71192 topics, and evaluate claims generated for 96 novel topics.

In this work, we assess the contribution of context to the quality of generated claims. In Durmus et al. (2019), context is defined as the path from a thesis (topic) node to a leaf (claim) node in an argument tree. In this work, however, we consider only arguments of depth 1 , directly addressing the topic, and leave context of larger depth to future work.

Additionally, for development and evaluation we use human annotations alongside automatic measures, aiming to answer nuanced questions - is it plausible that the claims be asserted by a human? do the generated claims tend to be opinions or factual? and, when they are the latter, do they tend to be factually true?

\section{Experimental Details}

\subsection{Data}

We compare the performance of fine-tuning GPT-2 on three argument datasets, two publicly available and one proprietary.

Rank-30k. This dataset includes $30 k$ arguments for 71 topics, labeled for their quality (Gretz et al., 2020). For fine-tuning GPT- 2 we consider all arguments with quality score (denoted there as WAscore) $>0.9$, resulting in 10,669 arguments. These arguments are typically 1-2 sentences long.

CE2.3k. This dataset consists of $2.3 k$ manually curated claims extracted from Wikipedia for 58 topics (Rinott et al., 2015). These claims are usually sub-sentence, concise phrases. We exclude claims for topics which are part of our dev set (see below). Further, we "wikify" each topic, i.e., automatically map each topic to a corresponding Wikipedia title (Shnayderman et al., 2019), and remove topics for which no such mapping is found. After this filtering, we remain with 1,489 claims for 29 topics.

LN55k. This proprietary dataset consists of 55,024 manually curated claims for the 192 topics in the train set of Ein-Dor et al. (2020). These claims were extracted from a corpus of some 400 million newspaper articles provided by LexisNexis, ${ }^{2}$ as done in Ein-Dor et al. (2020) for evidence rather than claims.

Whereas fine-tuning is done on varied datasources, for evaluation we focus on the dev and test topics from Ein-Dor et al. (2020). We exclude from both sets topics that are present in the Rank$30 \mathrm{k}$ dataset, resulting in a dev set of 35 topics and test set of 96 topics (see Appendix).

Throughout this work, we consider debatable topics which correspond to a single Wikipedia title, phrased as a suggestion for a policy - e.g., We should increase the use of telemedicine, or as a valuation analysis - e.g., telemedicine brings more harm than good.

${ }^{2}$ https://www. lexisnexis.com/en-us/home. page 


\subsection{Model}

For all experiments we fine-tune the medium-size GPT-2-355M model (Radford et al., 2019), utilizing the gpt-2-simple library. ${ }^{3}$ In order for the model to condition on topics, we represent each (topic, claim) pair from the training data as a single sequence, separated by a delimiter. In generation, the model is provided with a prompt in the form of a topic followed by a delimiter. We used top- $k$ truncation with $k=40$ and a conservative temperature of 0.7 , to accommodate a more readable, coherent output, while maintaining a level of creativity. We leave exploring other sampling techniques (e.g., Holtzman et al. (2019)) to future work. We restricted the length of each generated text to 50 BPE tokens, as preliminary experiments showed that very few GTs were longer. In addition, GTs were cleaned by removing non-ascii characters, parenthesis, single quotation marks, and some other erroneous symbols.

\subsection{Automatic Evaluation}

For evaluation, we consider perplexity and prefix ranking accuracy (Fan et al., 2018), considering the claims extracted by Ajjour et al. (2019) alongside their listed topics. ${ }^{4}$ For prefix ranking accuracy we condition each such claim on its real topic, as well as on 9 other random topics, and compute the fraction of times where conditioning on the real topic yields the highest probability by the finetuned model. For both evaluation measures, we report statistics for 10 samples of 100 claims sampled uniformly. Importantly, this dataset is independent of all the ones examined here, and so presumably not biased in favor of any of them. Due to the difference in style and topics from the training sets, the fine-tuned models may exhibit high perplexity, so it should be taken as a comparative measure, rather than an absolute one.

In addition, we evaluate the GTs by their quality and stance scores. For obtaining a quality score, we fine-tune BERT (Devlin et al., 2018) on Rank-30k, as in Gretz et al. (2020). This score aims to capture how well the output is written, giving preference to grammar, clarity and correct spelling. For obtaining a stance score, we utilize a proprietary internal

\footnotetext{
${ }^{3}$ https://github.com/minimaxir/ gpt-2-simple

${ }^{4}$ This dataset contains 12,326 claims from 465 topics extracted from debatepedia.org. We rephrase topics therein to fit our phrasing by adding the text "We should support" before of the listed topic.
}

service, based on a BERT model fine-tuned over the LN55k claims which were manually labeled for stance (Bar-Haim et al., 2017). A positive score indicates that a claim supports the topic, a negative score that it contests it, while a score close to zero suggests no clear stance. Since we are only interested in whether or not a sentence has a clear stance, we take the absolute value of the score. For both scores, we report statistics for 10 samples of 100 GTs sampled uniformly from the respective set.

\subsection{Annotation Tasks}

To further assess the quality of GTs we annotate their plausibility and stance. We do this in a cascade - only GTs considered plausible are subsequently annotated for their stance. The motivation for these two tasks is that together they enable us to assess the "claimness" of GTs, i.e., to determine to what extent the GTs represent coherent claims, relevant to the given topic. We used the Appen crowd-sourcing platform, ${ }^{5}$ with 7 annotators to annotate each GT. To control for annotation quality, we included hidden test questions, comprised of previously annotated rows with high confidence. Annotations by annotators with low accuracy on the test questions were removed (below $75 \%$ for plausibility and $80 \%$ for stance). Further, we relied on a channel of annotators which performed well on previous related tasks. For each task, we report inter-annotator agreement defined as the average Cohen's Kappa of annotators which have at least 50 common judgements with at least 5 other annotators.

Plausibility. In this task, given the GT only, without the context of its respective topic, the annotator should determine if it is plausible that a human would make this claim, considering grammar, coherence, and general "common sense". This task can be considered an extension of the readability task that is usually used to evaluate the quality of generated text (e.g., Beers and Nagy (2009)), while further asking to utilize common knowledge to judge that the content itself makes sense. For example, in the GT making blood donation free will help promote primary care, the notion of making blood donation free does not make sense as it is a voluntary act, hence the GT should be deemed implausible. A GT is considered plausible if $\geq 70 \%$ of the annotators considered it as such. The aver-

\footnotetext{
${ }^{5}$ wWw appen. com
} 
age inter-annotator Cohen's Kappa obtained in this task is 0.37 , which is common for such a subjective task (see, e.g., Ein-Dor et al. (2020) and Boltuzic and Snajder (2014)).

Stance. In this task we presented the annotators with GTs that were considered plausible, together with their respective topics. Annotators were asked to determine if the text supports the topic, contests it, or does not have a stance towards it. The label of the GT is determined by the majority vote, and if there is no majority label, it is considered as having no stance. As in the automatic measure of stance, we are mainly interested in evaluating if a GT bears any stance towards the topic, thus we consider both supports and contests labels as positives when reporting stance. The average interannotator Cohen's Kappa obtained in this task is 0.81 .

Table 2 shows examples of three types of labeled GTs - plausible and stance-bearing; plausible with no stance; and implausible. The results of these annotation tasks are made available as part of this work. ${ }^{6}$ The complete annotation guidelines are shared in the Appendix.

\section{Initial Generation}

Our first question was to examine the impact of the data used for fine-tuning GPT-2, aiming to identify an effective model that relies on publicly available data, and a presumably superior one that further relies on proprietary data of a much larger size.

Publicly available data. We considered Rank-30k alone, and combined with CE2.3k. We fine-tuned GPT- 2 for $2 k$ steps on the former, and $4 k$ steps on the latter. We denote the obtained models GPTRank and GPT-Rank-CE, respectively.

Proprietary data. We considered LN55k alone, as well as combined with all publicly available data. We fine-tuned GPT- 2 for $8 k$ steps on both. We denote the obtained models GPT-LN and GPTALL, respectively. ${ }^{7}$

For each of the 4 models we generated a total of 175 GTs, 5 conditioned on each of the $35 \mathrm{dev}$ topics. Note that the models are fine-tuned on datasets containing both supporting and contesting arguments, thus they may generate GTs of both stances

\footnotetext{
${ }^{6}$ https: / / www . research.ibm.com/haifa/ dept/vst/debating_data.shtml

${ }^{7}$ In section 8 , we describe the retrieval of $4.5 k$ (ostensible) claims from Wikipedia using a proprietary Claim Retrieval server. These claims are included in GPT-ALL.
}

as well. The manual and automatic evaluation of these GTs is described next.

As seen in Table 1 both proprietary models fine-tuned on much larger datasets - yield more plausible and stance-bearing GTs than their counterparts.

Among the proprietary-based models, while GPT-ALL has an advantage in plausibility, perplexity, and prefix ranking accuracy, GPT-LN is better when considering the ratio of GTs which are both plausible and stance-bearing - with $68 \%(119 / 175)$ such GTs, compared to $62.3 \%$ (109/175) for GPT2-ALL. It seems that adding more data, varied in type and style, could negatively impact the relevance and usefulness of GTs. Thus, we choose GPT-LN as the model to utilize for subsequent experiments.

As for the publicly-based models, GPT-Rank$\mathrm{CE}$ has a small advantage in plausible and stancebearing GTs, compared to GPT-Rank. However, the performance of the latter is typically much better in the automatic measures. Especially, we note the advantage in predicted quality - as expected, generated arguments from the GPT-Rank model have higher quality, as both this model and the argument quality model were trained on a similar type of data. However, when adding the CE2.3k dataset to the training set, the quality of GTs declines. Thus, even though the differences between the two models are overall not substantial, we choose GPTRank for subsequent experiments.

It should be noted that there is a clear difference between the GTs of GPT-LN and GPT-Rank, as evident in Table 2. The former are short (12.4 tokens on average), and may contain utterances with as few as 3-4 tokens (as in the GT in row 3). By contrast, GTs generated by GPT-Rank contain 23 tokens on average, and 22/175 of them contain at least two sentences (as in the GT in row 4). In addition, shorter GTs tend to be plausible - on average, plausible GTs from GPT-LN have 12.1 tokens, compared to 15.4 tokens for implausible GTs. Likewise, plausible GTs from GPT-Rank contain 20.5 tokens, on average, compared to 26 tokens for implausible GTs.

We note that for all models, the predicted quality and stance strength are only slightly lower than their counterpart measures on the training set, suggesting that generation tends to maintain these values. 


\begin{tabular}{|c|c|c|c|c|c|c|c|c|}
\hline & PL & PL + ST & PPL & PR & P-QU & P-ST & P-QU* & P-ST* \\
\hline GPT-LN & $75.4 \%$ & $\mathbf{6 8 \%}$ & 188.9 & 0.69 & 0.75 & 0.99 & 0.78 & 1.00 \\
\hline GPT-ALL & $78.9 \%$ & $62.3 \%$ & 82.7 & 0.74 & 0.76 & 0.97 & 0.79 & 1.00 \\
\hline GPT-Rank & $53.1 \%$ & $51.4 \%$ & 150.8 & 0.75 & 0.85 & 0.99 & 0.85 & 1.00 \\
\hline GPT-Rank-CE & $64.6 \%$ & $\mathbf{5 4 . 9 \%}$ & 388.4 & 0.65 & 0.8 & 0.98 & 0.84 & 1.00 \\
\hline
\end{tabular}

Table 1: Results on the dev set of models fine-tuned on proprietary (top 2) and publicly available (bottom 2) data sources. $\mathrm{PL}=$ ratio of plausible claims, $\mathrm{PL}+\mathrm{ST}=$ ratio of plausible and stance bearing claims, $\mathrm{PPL}=$ perplexity, $\mathrm{PR}=$ prompt ranking accuracy, $\mathrm{P}-\mathrm{QU}=$ predicted quality, $\mathrm{P}-\mathrm{ST}=$ predicted (absolute) stance. Asterisk indicates values for the training set.

\begin{tabular}{|l|l|l|l|}
\hline Topic & GT & Model & Label \\
\hline $\begin{array}{l}\text { We should abandon } \\
\text { democracy }\end{array}$ & A proper democracy is good for the country & $\begin{array}{l}\text { GPT-LN } \\
\text { Plausible } \\
\text { hand }\end{array}$ & $\begin{array}{l}\text { Plausible } \\
\text { no stance }\end{array}$ \\
\hline $\begin{array}{l}\text { We should lower the } \\
\text { drinking age }\end{array}$ & the age of majority in the country was lowered to 18 \\
\hline $\begin{array}{l}\text { We should ban free } \\
\text { newspapers }\end{array}$ & free newspapers reduce crime & GPT-LN \\
\hline $\begin{array}{l}\text { We should increase } \\
\text { government regula- } \\
\text { tion }\end{array}$ & $\begin{array}{l}\text { we need regulation to make sure our country is protected. } \\
\text { with more government involvement in our daily lives, busi- } \\
\text { nesses can hire more workers and produce more output. }\end{array}$ & GPT-Rank & $\begin{array}{l}\text { Plausible } \\
\text { has stance }\end{array}$ \\
\hline $\begin{array}{l}\text { We should fight for } \\
\text { Palestinian indepen- } \\
\text { dence }\end{array}$ & $\begin{array}{l}\text { the liberation of Palestine will be impossible if the Palestini- } \\
\text { ans are ruled by corrupt Israeli and Palestinian governments }\end{array}$ & GPT-Rank & $\begin{array}{l}\text { Plausible } \\
\text { no stance }\end{array}$ \\
\hline $\begin{array}{l}\text { We should ban lot- } \\
\text { teries }\end{array}$ & $\begin{array}{l}\text { lotteries are a great way for children to learn about different } \\
\text { cultures and find similar things to do }\end{array}$ & GPT-Rank & Implausible \\
\hline
\end{tabular}

Table 2: Examples of GTs generated by the GPT-LN and GPT-Rank models, labeled for plausibility and stance.

\section{Adding context}

Can we improve GTs by conditioning their generation on more context? To evaluate this hypothesis we considered two context variations, one in which we frame the topic and the other in which we frame the claim.

Framing the topic. We prepend to the topic the first sentence from the Wikipedia page describing the topic, to explore whether this added knowledge could guide models to generate more relevant and meaningful GTs. The motivation for selecting the first sentence from Wikipedia is to provide the model a concise guidance towards the respective topic via the main terms it may relate to, which usually appear in the first Wikipedia sentence. The relevant Wikipedia page is found by Wikifying the topic, as described in $\S 3.1$.

Framing the claim. We also tried to append to the topic a short sentence describing an aspect relevant to discussing it, hypothesizing that adding a concrete aspect will guide the generation process in that direction. Unfortunately, this did not work well, and details are deferred to the appendix.

Evaluation: We fine-tune GPT-2 from scratch on the modified training data of Rank-30k and LN55k and refer to the new models as GPT-Rank-FWS,
GPT-LN-FWS (First Wikipedia Sentence, when framing the topic). We generate a sample of 5 (GPT-Rank-FWS) or 10 (GPT-LN-FWS) GTs per dev topic.

Results: Table 3 presents the results for the FWS models. For both FWS models the perplexity has improved, as well as the plausibility of GTs, presumably, since the added context helps to avoid some illogical phrases. For example, the GT The human condition is the greatest human achievement for the topic We should subsidize the human mission to Mars which was generated by GPT-LN was considered implausible, whereas all GTs for this topic generated by GPT-LN-FWS were considered plausible. After stance labeling, the advantage of GPT-LN-FWS remains, while GPT-RankFWS performs slightly worse. In addition, the GPT-Rank-FWS is slightly worse in predicted quality and stance. Thus, for further experiments, we chose the GPT-LN-FWS and GPT-Rank models.

\section{Factual, Opinion, and Generic Claims}

An interesting facet when considering argumentative claims, is whether they attempt to convey facts, or rather personal opinions. Thus, we explored if GTs generated by our two models are characterized as more factual or opinionated. Further, 


\begin{tabular}{|c|c|c|c|c|c|c|}
\hline & PL & PL + ST & PPL & PR & P-QU & P-ST \\
\hline GPT-LN & $76.6 \%$ & $66.3 \%$ & 188.9 & 0.69 & 0.75 & 0.99 \\
\hline GPT-LN-FWS & $85.1 \%$ & $\mathbf{7 3 . 1 \%}$ & 88.6 & 0.74 & 0.76 & 0.99 \\
\hline GPT-Rank & $53.1 \%$ & $\mathbf{5 1 . 4 \%}$ & 150.8 & 0.75 & 0.85 & 0.99 \\
\hline GPT-Rank-FWS & $58.9 \%$ & $49.7 \%$ & 71.6 & 0.76 & 0.83 & 0.97 \\
\hline
\end{tabular}

Table 3: Results on the dev set of models with and without conditioning on the first sentence of the Wikipedia page corresponding to the topic. Column titles as in Table 1. For GPT-Rank we used 175 GTs as per Section 4. For GPT-LN, data includes an additional 175 GTs. Hence, numbers here differ from Table 1.

given growing concern over misuse of language models such as GPT-2 to spread fake news and misinformation (Zellers et al., 2019; Solaiman et al., 2019), we assessed the truth value of GTs deemed factual. For this purpose, we first sampled 200 plausible and stance-bearing GTs each generated by GPT-LN-FWS and GPT-Rank, respectively, and annotated all 400 GTs for being an opinion or (ostensibly) factual, using the Appen platform, and relying on similar annotation controls as described in $\S 3.4$. The results of this annotation task are made available as part of this work, and the annotation guidelines are shared in the Appendix. The average inter-annotator agreement was 0.25 .

When considering labels with a majority vote of at least $70 \%, 70$ of the GTs generated by GPT-Rank are considered factual and 63 opinion, as opposed to 46 and 105 of those generated by GPT-LN-FWS, respectively. A possible explanation is that Rank30k claims - on which GPT-Rank was fine-tuned tend to be more elaborate and explanatory, describing a cause and effect that correspondingly yields more factual GTs; e.g., the GT genetic engineering can help further scientific developments in cancer treatment, as well as improve the long term prognosis of such diseases as help maintain a safe and effective regulatory regime for their development, for the topic We should further exploit genetic engineering. By contrast, LN55k claims are often short and concise, and perhaps more prone to express the journalist opinion; hence, training on these data yields more opinionated GTs, e.g., the "sex" revolution has failed or the gender pay gap is unfair. Indeed, the average number of tokens in factual GTs is 17.3 , compared to 14.2 for opinion GTs.

Next, we aimed to assess whether factual GTs are indeed true. A random sample of 23 and 40 factual GTs generated by GPT-LN-FWS and GPTRank, respectively, were labeled for their truth value by a professional debater experienced in this task, that also was asked to assess whether the "fake facts" were nonetheless common in contemporary discourse.

Of the 23 GPT-LN-FWS GTs, 13 were considered true, the others being a mix of false or nonfactual GTs. The true GTs include some simple, almost trivial statements such as Speed limits are designed to help reduce road fatalities, or more evidence-based facts such as rat poisons have been linked to the development of Parkinson's disease, Alzheimer's disease and migraines. Among the 4 false GTs, it is interesting, albeit perhaps unsurprising, to find that 2 were marked as common in discourse: Flu vaccinations are associated with higher rates of adverse drug reactions and serious health complications, and poly-amorous relationships are linked to higher levels of sexual risk.

For the 40 GPT-Rank factual GTs, 21 were deemed true. Overall, the ratio of true GTs is similar to that of GPT-LN-FWS GTs. It seems that some of the other GTs are mixed, characterized by opening with an opinionated statement, which is followed by a factual claim, e.g., we should not abandon chain stores (Opinion) as they provide a steady supply of goods and services to the community (True fact). One of the 3 false GTs could be considered common in discourse, the alternative vote would cause voters to be disenfranchised.

The aforementioned short GTs suggested that GTs tend to be rather generic, in the sense that stating that something "has failed" or "is unfair", can be done (coherently) for a great variety of contexts. Indeed, such GTs are reminiscent of those generated by Bilu and Slonim (2016). To assess to what extent such GTs are generic, we sampled 100 of them, and annotated them ourselves. In this sample, 54 of the GTs were deemed generic, suggesting that such GTs are prevalent, but by no means the only types of texts being generated.

\section{The Complete Pipeline}

\subsection{Ranking Generated Claims}

So far we have assessed the overall ability of the models to generate relevant claims. A natural ques- 
tion is whether one can efficiently rank the obtained GTs, retaining only the most attractive ones for downstream tasks. This could be considered somewhat analogous to Claim Retrieval tasks, where first a large amount of argument candidates is retrieved, and are then ranked according to their relevance (e.g., Levy et al. (2014); Stab et al. (2018); Ein-Dor et al. (2020)).

We considered three existing models for ranking GTs - the argument quality and stance models described in $\S 3.3$, and a Claim Detection (CD) proprietary service, obtained by training a BERT model on LN55k. The data for training the model is augmented with negative samples from the same corpus - sub-sentential fragments which were labeled as non-claims. The objective of the model is to differentiate between claims and non-claims, and is similar to that described in Ein-Dor et al. (2020) for Evidence detection. For evaluation we considered GTs generated on the dev set by GPT-Rank and GPT-LN-FWS for which we had a definite label for relevance to the topic. Specifically, GTs which were annotated as "implausible" by a majority of annotators were assigned a label of 0 . GTs which were annotated as plausible, and then annotated for stance, were labeled according to the latter annotation: 1 if they were annotated as Pro or $C o n$, and 0 otherwise. In total, we considered 211 positive and 120 negative GTs.

Overall, the CD score is best correlated with the labels - Pearson's $\rho=0.41$, compared to 0.12 for (absolute) stance, and 0.01 for argument quality. In addition, we ranked the GTs within each topic w.r.t each score, and calculated the ratio between the number of positives in the top 3 and bottom 3 . As before, $\mathrm{CD}$ is preferred, with $81 / 40$ positives in the top/bottom, compared to $70 / 56$ (stance) and $71 / 67$ (argument quality). See a short discussion about this result in the Appendix.

Accordingly, we defined the generation pipeline as follows: (i) Fine-tune GPT-2 to obtain GPTRank (Model-1) or GPT-LN-FWS (Model-2); (ii) Generate with the topic as a prompt (Model-1), or prepend the - automatically extracted - first sentence of the associated Wikipedia article to the topic and use the resultant text as a prompt; (iii) rank the obtained GTs according to their CD score. In principle, one could set a strict threshold on the $\mathrm{CD}$ score, and generate a large number of texts until a sufficient number pass this threshold. We plan to investigate this direction in future work.

\begin{tabular}{|c|c|c|c|c|}
\hline & PL & PL + ST & P-QU & P-ST \\
\hline GPT-LN-FWS & $82.4 \%$ & $79.5 \%$ & 0.78 & 0.97 \\
\hline GPT-Rank & $58.8 \%$ & $57 \%$ & 0.85 & 0.98 \\
\hline
\end{tabular}

Table 4: Results on the test set of the GPT-LN-FWS and GPT-Rank models, with ranking using the claim detection model. Column titles as in Table 1.

\subsection{Test Set Results}

With the above pipeline, we now proceed to generate 20 GTs for each of the 96 topics in the test set, using the GPT-LN-FWS and GPT-Rank models. We then take the top 7 GTs according to the CD score, per topic, resulting in 672 GTs overall for each model. As done for the dev set, we label these GTs for plausibility and stance, as well as calculate their predicted quality and stance.

Results are presented in Table 4. The overall ratio of GTs perceived as both plausible and carrying stance for the GPT-LN-FWS model and the GPT-Rank model are $79.5 \%$ and $57 \%$, respectively, conveying the advantage of fine-tuning on much larger data (see the appendix for examples). In addition, our test set results echo the results obtained on the dev set, suggesting that our analysis on the dev set is relevant for the test set as well, and that our models generalize well to unseen topics.

\section{Claim Generation vs. Claim Retrieval}

Given a controversial topic, Claim Generation and Claim Retrieval both aim to provide claims pertaining to it. It is therefore interesting to understand the interplay between the two tasks. Specifically, thinking of Claim Generation as a mean to augment the output of Claim Retrieval, we ask whether GTs tend to be novel, or a repetition of retrieved claims, and how does the quality of the two compare. In addition, we explore how Claim Retrieval can facilitate the training of the Claim Generation pipeline suggested in this work.

How novel are the generated claims? Similar to the manually-curated claims of the LN55k dataset, we also had access to such claims pertaining to $34 / 35$ topics in the dev set (henceforth, the LN claims). For comparison we used 169 GTs (5 per topic, one duplicate removed) from the GTs generated by GPT-LN for these 34 topics (see Section $\S 4$ ). To measure similarity between GTs and LN claims we fine-tuned BERT on a Semantic Text Similarity benchmark (Cer et al., 2017). The resultant model was used to find for each GT the top 
matching LN claim. Manual examination suggests that a score of 0.75 roughly differentiates pairs with semantically similar claims and those which are not (Table 5). Note that semantically similar claims may still have opposing stance, but in this case we also consider the GT as appearing in the corpus (in its negated form).

Taking all pairs with score $\geq 0.75$, we get that only 20/169 of the GTs have a semanticallysimilar counterpart among the LN claims, suggesting that GTs tend to be novel. Moreover, we see that the match score is well correlated with the number of annotators who labeled a GT as plausible (Pearson's $\rho=0.31$ ) or as having a stance ( $\rho=0.47)$. Similarly, in general, $127 / 169$ GTs were determined by human annotators to be plausible and $114 / 169$ as having a stance. In comparison, $19 / 20$ GTs with match score $\geq 0.75$, were deemed both plausible and as having a stance. This suggests, as may be expected, that GTs are more likely to represent valid claims if they already appear in some phrasing within a human-authored corpus. Future work might use this to validate GTs, or, conversely, to guide claim retrieval.

How good are the generated claims? Having matched GTs to "real" claims allows us to compare not only their novelty, but also their quality. Namely, for each of the 169 pairs we asked crowd annotators which of the two claims "would have been preferred by most people to discuss the topic?", using the same process as in section $\S 3$. Among these pairs, in 41 cases both claims appeared to be similarly good (a $3: 4$ split); in 57 the GT is preferred; and in 71 the $\mathrm{LN}$ claim is considered better. Among the 20 pairs which are highly similar, in 4 both claims are equally good, in 13 the GT is better and in 4 the $\mathrm{LN}$ claim is preferred. Thus, at least in this small sample, when the two claims are conveying a similar message, human annotators seem to prefer the GPT-2 version over the human authored one.

Can claim retrieval facilitate generation? The suggested pipeline assumes access to a dataset of actual claims to fine-tune GPT-2. However, initial analysis suggest that even with no a-priory labeled data, having access to a high quality Claim Retrieval engine, can be enough to facilitate Claim Generation. Using a propriety Claim Retrieval server, we first query Wikipedia to retrieve sentence candidates, in a similar process to that described in Ein-Dor et al. (2020) for retrieving Evidence candidates. We then rank them according to the Claim Detection model described in §7.1. Overall, we obtain 4427 (ostensible) claims from Wikipedia for the 192 train topics. We fine-tuned GPT-2 on them, and evaluated the results as done for the other datasets (§4). Since these data are not manually curated, some of the texts used for fine-tuning are not actual claims. Nonetheless, human annotators deemed 124/175 GTs as plausible; average perplexity is 264 , mean prefix ranking accuracy is 0.61 , and average argument quality is 0.75 . These results are comparable to those obtained over the much larger Rank-30k dataset, suggesting that a good solution to the Claim Retrieval task embodies a good solution to the Claim Generation task.

\section{Further observations}

What characterizes implausible GTs? We considered the 51 GPT-LN-FWS test-set GTs which were deemed implausible. More than half seem to contradict common sense, often by connecting pairs of unrelated terms as in the titular the workweek is the best time to start a family, for the topic We should increase the workweek; or via connecting related terms in an odd manner as in LGBT adoption is a critical component of a child's life for the topic We should legalize LGBT adoption. Other reasons for implausibility include weird phrasings (e.g., the housing in public housing is disastrously unaffordable) and bad grammar (e.g., that the benefits of the MRT network outweigh its costs).

COVID-19 debates. Our pipeline relies heavily on the massive pre-training of GPT-2, that naturally included sentences pertaining - at least to some extent - to topics in our dev and test sets. It is therefore interesting to examine the GTs obtained for topics which were presumably less abundant in the pre-training data. Hence, while sheltering at home, we have generated 20 GTs for each of the following two topics: We should subsidize the COVID-19 drug development and Coronavirus face masks should be mandatory using the GPT-LNFWS model. For the first topic, only 4 of the 20 GTs were coherent and relevant, while many of the others talked about HIV, alluded to the opioid crisis, or were outright absurd - the use of artificial sweeteners in food should be a crime. The four "good" ones were of generic form, yet some showed an ability to extrapolate to relevant terms, without them being mentioned explicitly in the prefix. For example, in the GT the COVID-19 vaccine will 


\begin{tabular}{|c|l|l|c|}
\hline & Generated claim & Matched claim & Score \\
\hline 1 & natural gas has positive effects on the environment & natural gas can have a negative environmental effect & 0.85 \\
\hline 2 & $\begin{array}{l}\text { alternative medicine could be a good option for } \\
\text { some patients }\end{array}$ & alternative medicine could be useful & 0.76 \\
\hline 3 & the lottery could drive away investment & lottery could be a significant source of revenue & 0.75 \\
\hline 4 & $\begin{array}{l}\text { lower retirement ages would promote more } \\
\text { long-term job stability }\end{array}$ & $\begin{array}{l}\text { a higher minimum retirement age would lead to people } \\
\text { working longer translating in greater economic output }\end{array}$ & 0.74 \\
\hline
\end{tabular}

Table 5: Examples of matching of generated claims to manually-curated claims.

be a very effective vaccine as compared to other vaccines, while "COVID-19" and "vaccine" are mentioned separately in the prefix (i.e., in the first sentence of the Wikipedia page COVID-19 drug development), the term "COVID-19 vaccine" is not For the second topic, 12 of the GTs are coherent and relevant, presumably because the use of face masks to prevent disease is more general, and may have have been discussed in the pre-training data. It has probably been true of previous airborne viruses that, for example, the use of face masks is the best way to keep people safe. Among the irrelevant GTs there is mention of other medical conditions, such as Ebola, diarrhoea and mosquito bites. The full list of GTs for these two topics, as well as 3 additional ones, are made available as part of this work.

\section{Conclusions}

We suggest a claim-generation pipeline, based on a fine-tuned GPT- 2 model augmented by framing the topic, and filtered using Claim Detection tools. Results on a diverse set of 96 new topics demonstrate the merit of our approach. As expected, fine tuning on a larger dataset of claims leads to more accurate generation. Yet, the coherency of the dataset also matters; simple merging of datasets of different flavors does not improve generation, and may even hamper it.

To evaluate the generation models we examined several measures, which roughly estimate how "good" the generated text is. But since they do so from different perspectives, they are often not consistent with one another (Wachsmuth et al., 2017). Here they were combined heuristically, but future work should explore this more rigorously.

Our work highlights some of the relations between Claim Generation, Claim Retrieval, and Claim Detection. In our pipeline, Claim Detection is used to weed out poorly-generated claims. Further, we show that Claim Retrieval is a sufficient basis - alongside a powerful language model - for building a claim generation pipeline; and that
Claim Generation may augment Claim Retrieval with additional novel claims.

Here, GPT-2 was used with a "default" setting. However, there is clearly an interesting trade-off between creativity and coherence, and balancing the two to fit an intended use case - perhaps even interactively - which we intend to explore in future research.

Finally, the claims generated by our pipeline display both subjective opinions and factual assertions. In the latter case, our initial analysis indicates that the generated claims of a factual nature are often, but certainly not always, factually true. Thus, our work highlights a new emerging front in the rapidly expanding area of fact verification - that of distinguishing valid factual statements from non-valid ones, on top of automatically generated texts.

\section{Ethical note}

Argument generation has the potential of being misused (Solaiman et al., 2019), as it can potentially allow to automatically generate a variety of false assertions regarding a topic of interest. In addition, GPT-2 text generations have been shown to exhibit different levels of bias towards different demographics (Sheng et al., 2019). Nonetheless, the way to address these dangers is for the community to recognize and better understand the properties of such generated texts, and we hope this work provides a step forward in this direction. As, to the best of our knowledge, this is the first work leveraging GPT-2 in the context of argumentation, such work can be used to advance research in the argument generation community, by surfacing issues of such systems. Furthermore, in our setting we allow for arguments to be generated on both sides of the topic, thus if one side is misrepresented, it would be easily uncovered.

\section{References}

Yamen Ajjour, Milad Alshomary, Henning Wachsmuth, and Benno Stein. 2019. Modeling frames in ar- 
gumentation. In Proceedings of the 2019 Conference on Empirical Methods in Natural Language Processing and the 9th International Joint Conference on Natural Language Processing (EMNLPIJCNLP), pages 2922-2932, Hong Kong, China. Association for Computational Linguistics.

Ateret Anaby-Tavor, Boaz Carmeli, Esther Goldbraich, Amir Kantor, George Kour, Segev Shlomov, Naama Tepper, and Naama Zwerdling. 2019. Not enough data? deep learning to the rescue!

Roy Bar-Haim, Indrajit Bhattacharya, Francesco Dinuzzo, Amrita Saha, and Noam Slonim. 2017. Stance classification of context-dependent claims. In Proceedings of the 15th Conference of the European Chapter of the Association for Computational Linguistics: Volume 1, Long Papers, pages 251-261, Valencia, Spain. Association for Computational Linguistics.

Scott F Beers and William E Nagy. 2009. Syntactic complexity as a predictor of adolescent writing quality: Which measures? which genre? Reading and Writing, 22(2):185-200.

Yonatan Bilu, Ariel Gera, Daniel Hershcovich, Benjamin Sznajder, Dan Lahav, Guy Moshkowich, Anael Malet, Assaf Gavron, and Noam Slonim. 2019. Argument invention from first principles. In Proceedings of the 57th Annual Meeting of the Association for Computational Linguistics, pages 10131026. Association for Computational Linguistics.

Yonatan Bilu and Noam Slonim. 2016. Claim synthesis via predicate recycling. In Proceedings of the 54th Annual Meeting of the Association for Computational Linguistics (Volume 2: Short Papers), pages 525-530, Berlin, Germany. Association for Computational Linguistics.

Filip Boltuzic and J. Snajder. 2014. Back up your stance: Recognizing arguments in online discussions. In ArgMining@ACL.

Giuseppe Carenini and Johanna D Moore. 2006. Generating and evaluating evaluative arguments. Artificial Intelligence, 170(11):925-952.

Daniel Cer, Mona Diab, Eneko Agirre, Inigo LopezGazpio, and Lucia Specia. 2017. Semeval-2017 task 1: Semantic textual similarity-multilingual and cross-lingual focused evaluation. Eleventh International Workshop on Semantic Evaluations.

Jacob Devlin, Ming-Wei Chang, Kenton Lee, and Kristina Toutanova. 2018. Bert: Pre-training of deep bidirectional transformers for language understanding. arXiv preprint arXiv: 1810.04805 .

Esin Durmus, Faisal Ladhak, and Claire Cardie. 2019. The role of pragmatic and discourse context in determining argument impact. In Proceedings of the 2019 Conference on Empirical Methods in Natural Language Processing and the 9th International Joint Conference on Natural Language Processing
(EMNLP-IJCNLP), pages 5668-5678, Hong Kong, China. Association for Computational Linguistics.

Liat Ein-Dor, Eyal Shnarch, Lena Dankin, Alon Halfon, Benjamin Sznajder, Ariel Gera, Carlos Alzate, Martin Gleize, Leshem Choshen, Yufang Hou, et al. 2020. Corpus wide argument mining-a working solution. In Proceedings of the Thirty-Fourth AAAI Conference on Artificial Intelligence.

Roxanne El Baff, Henning Wachsmuth, Khalid Al Khatib, Manfred Stede, and Benno Stein. 2019. Computational argumentation synthesis as a language modeling task. In Proceedings of the 12th International Conference on Natural Language Generation, pages 54-64, Tokyo, Japan. Association for Computational Linguistics.

Angela Fan, Mike Lewis, and Yann Dauphin. 2018. Hierarchical neural story generation. In Proceedings of the 56th Annual Meeting of the Association for Computational Linguistics (Volume 1: Long Papers), pages 889-898, Melbourne, Australia. Association for Computational Linguistics.

Shai Gretz, Roni Friedman, Edo Cohen-Karlik, Assaf Toledo, Dan Lahav, Ranit Aharonov, and Noam Slonim. 2020. A large-scale dataset for argument quality ranking: Construction and analysis. In Proceedings of the Thirty-Fourth AAAI Conference on Artificial Intelligence.

Christopher Hidey and Kathy McKeown. 2019. Fixed that for you: Generating contrastive claims with semantic edits. In Proceedings of the 2019 Conference of the North American Chapter of the Association for Computational Linguistics: Human Language Technologies, Volume 1 (Long and Short Papers), pages 1756-1767, Minneapolis, Minnesota. Association for Computational Linguistics.

Ari Holtzman, Jan Buys, Li Du, Maxwell Forbes, and Yejin Choi. 2019. The curious case of neural text degeneration.

Xinyu Hua, Zhe Hu, and Lu Wang. 2019. Argument generation with retrieval, planning, and realization. In Proceedings of the 57th Annual Meeting of the Association for Computational Linguistics, pages 2661-2672, Florence, Italy. Association for Computational Linguistics.

Xinyu Hua and Lu Wang. 2018. Neural argument generation augmented with externally retrieved evidence. In Proceedings of the 56th Annual Meeting of the Association for Computational Linguistics (Volume 1: Long Papers), pages 219-230, Melbourne, Australia. Association for Computational Linguistics.

Nitish Shirish Keskar, Bryan McCann, Lav R Varshney, Caiming Xiong, and Richard Socher. 2019. Ctrl: A conditional transformer language model for controllable generation. arXiv preprint arXiv:1909.05858. 
Ran Levy, Yonatan Bilu, Daniel Hershcovich, Ehud Aharoni, and Noam Slonim. 2014. Context dependent claim detection. In Proceedings of COLING 2014, the 25th International Conference on Computational Linguistics: Technical Papers, pages 14891500, Dublin, Ireland. Dublin City University and Association for Computational Linguistics.

Matan Orbach, Yonatan Bilu, Ariel Gera, Yoav Kantor, Lena Dankin, Tamar Lavee, Lili Kotlerman, Shachar Mirkin, Michal Jacovi, Ranit Aharonov, and Noam Slonim. 2019. A dataset of general-purpose rebuttal. In Proceedings of the 2019 Conference on Empirical Methods in Natural Language Processing and the 9th International Joint Conference on Natural Language Processing (EMNLP-IJCNLP), pages 55915601 , Hong Kong, China. Association for Computational Linguistics.

Alec Radford, Jeffrey Wu, Rewon Child, David Luan, Dario Amodei, and Ilya Sutskever. 2019. Language models are unsupervised multitask learners. OpenAI Blog, 1(8):9.

Paul Reisert, Naoya Inoue, Naoaki Okazaki, and Kentaro Inui. 2015. A computational approach for generating toulmin model argumentation. In Proceedings of the 2nd Workshop on Argumentation Mining, pages $45-55$.

Ruty Rinott, Lena Dankin, Carlos Alzate Perez, Mitesh M. Khapra, Ehud Aharoni, and Noam Slonim. 2015. Show me your evidence - an automatic method for context dependent evidence detection. In Proceedings of the 2015 Conference on Empirical Methods in Natural Language Processing, pages 440-450, Lisbon, Portugal. Association for Computational Linguistics.

Benjamin Schiller, Johannes Daxenberger, and Iryna Gurevych. 2020. Aspect-controlled neural argument generation. arXiv preprint arXiv:2005.00084.

Emily Sheng, Kai-Wei Chang, Premkumar Natarajan, and Nanyun Peng. 2019. The woman worked as a babysitter: On biases in language generation. In Proceedings of the 2019 Conference on Empirical Methods in Natural Language Processing and the 9th International Joint Conference on Natural Language Processing, EMNLP-IJCNLP 2019, Hong Kong, China, November 3-7, 2019, pages 34053410. Association for Computational Linguistics.

Ilya Shnayderman, Liat Ein-Dor, Yosi Mass, Alon Halfon, Benjamin Sznajder, Artem Spector, Yoav Katz, Dafna Sheinwald, Ranit Aharonov, and Noam Slonim. 2019. Fast end-to-end wikification.

Irene Solaiman, Miles Brundage, Jack Clark, Amanda Askell, Ariel Herbert-Voss, Jeff Wu, Alec Radford, Gretchen Krueger, Jong Wook Kim, Sarah Kreps, Miles McCain, Alex Newhouse, Jason Blazakis, Kris McGuffie, and Jasmine Wang. 2019. Release strategies and the social impacts of language models.
Christian Stab, Tristan Miller, Benjamin Schiller, Pranav Rai, and Iryna Gurevych. 2018. Crosstopic argument mining from heterogeneous sources. In Proceedings of the 2018 Conference on Empirical Methods in Natural Language Processing, pages 3664-3674, Brussels, Belgium. Association for Computational Linguistics.

Henning Wachsmuth, Nona Naderi, Ivan Habernal, Yufang Hou, Graeme Hirst, Iryna Gurevych, and Benno Stein. 2017. Argumentation quality assessment: Theory vs. practice. In Proceedings of the 55th Annual Meeting of the Association for Computational Linguistics (Volume 2: Short Papers), pages 250-255, Vancouver, Canada. Association for Computational Linguistics.

Henning Wachsmuth, Manfred Stede, Roxanne El Baff, Khalid Al-Khatib, Maria Skeppstedt, and Benno Stein. 2018. Argumentation synthesis following rhetorical strategies. In Proceedings of the 27th International Conference on Computational Linguistics, pages 3753-3765, Santa Fe, New Mexico, USA. Association for Computational Linguistics.

Douglas Walton and Thomas F Gordon. 2012. The carneades model of argument invention. Pragmatics \& Cognition, 20(1):1-31.

Rowan Zellers, Ari Holtzman, Hannah Rashkin, Yonatan Bisk, Ali Farhadi, Franziska Roesner, and Yejin Choi. 2019. Defending against neural fake news. In H. Wallach, H. Larochelle, A. Beygelzimer, F. d'Alché Buc, E. Fox, and R. Garnett, editors, Advances in Neural Information Processing Systems 32, pages 9054-9065. Curran Associates, Inc.

Ingrid Zukerman, Richard McConachy, and Kevin B Korb. 1998. Bayesian reasoning in an abductive mechanism for argument generation and analysis. In AAAI/IAAI, pages 833-838.

\section{A Examples of Labeled GTs}

Tables 6 and 7 detail GTs generated over the test set by the two models. Each table details two GTs which were labeled implausible, two that were labeled as plausible but lacking stance toward the topic prompt, and two that are coherent GTs for the topic.

\section{B Framing Claims}

In an attempt to frame the GTs, we append to the topic a short sentence describing an aspect related to the claim, hypothesizing that adding a concrete aspect will guide the generation process in that direction. We consider the aspects (or frames) appearing $\geq 100$ times in the dataset of Ajjour et al. (2019), and manually map each aspect to a related list of Wikipedia pages. Using Wikification, we keep in the training set only claims that reference 


\begin{tabular}{|l|l|l|l|}
\hline Topic & GT & Label & Comment \\
\hline We should close landfills & $\begin{array}{l}\text { landfills are not a sustainable way to fund } \\
\text { the nation's health }\end{array}$ & implausible & $\begin{array}{l}\text { The text is coherent. However, landfills were never in- } \\
\text { tended to provide revenue that will somehow contribute } \\
\text { to health funds, which makes this claim implausible. }\end{array}$ \\
\hline $\begin{array}{l}\text { Criticism of the Iraq War } \\
\text { is justified }\end{array}$ & $\begin{array}{l}\text { the Iraq War is a costly and costly mis- } \\
\text { take }\end{array}$ & implausible & $\begin{array}{l}\text { The odd repetition of the term "costly" is why this claim } \\
\text { is considered implausible. }\end{array}$ \\
\hline $\begin{array}{l}\text { We should nationalize the } \\
\text { health care industry }\end{array}$ & $\begin{array}{l}\text { The health care sector is one of the } \\
\text { fastest growth sectors of the economy. }\end{array}$ & no stance & $\begin{array}{l}\text { The claim is praising the effects of the health care indus- } \\
\text { try on the economy, thus suggesting to keep things as } \\
\text { they are. However, it could be also interpreted as some- } \\
\text { thing that governments could profit from, thus suggest- } \\
\text { ing to support the topic. }\end{array}$ \\
\hline $\begin{array}{l}\text { We should abandon } \\
\text { Valentine's day }\end{array}$ & $\begin{array}{l}\text { The Valentine's Day holiday is one of } \\
\text { the most misunderstood and misused hol- } \\
\text { idays. }\end{array}$ & $\begin{array}{l}\text { The attitude towards Valientine's Day can be understood } \\
\text { as negative, as it is misunderstood, or as positive, as } \\
\text { something that should be actually getting better respect } \\
\text { from the public. In addition, the use of the verb "mis- } \\
\text { used" is rather misused in this context. Either way, there } \\
\text { is no clear stance towards abandoning it. }\end{array}$ \\
\hline $\begin{array}{l}\text { We should disband the } \\
\text { Council Nations Security }\end{array}$ & $\begin{array}{l}\text { the United Nations Security Council is } \\
\text { an essential forum for maintaining the } \\
\text { international community's collective ef- } \\
\text { forts to fight terrorism }\end{array}$ & plausible and \\
has stance & & \\
\hline $\begin{array}{l}\text { We should subsidize } \\
\text { transportation network } \\
\text { companies }\end{array}$ & $\begin{array}{l}\text { networks in the country will help boost } \\
\text { economic growth, provide enhanced fuel } \\
\text { efficiency and reduce the use of private } \\
\text { vehicles }\end{array}$ & $\begin{array}{l}\text { plausible and } \\
\text { has stance }\end{array}$ & \\
\hline
\end{tabular}

Table 6: Examples of GTs generated by GPT-LN-FWS on the test set.

at least one of these Wikipedia pages. Finally, we manually phrase each aspect as a framing sentence, e.g., Consider how this relates to the economy for the Economy aspect, and append it to the topic separated by a delimiter.

For evaluation, we generated 15 GTs per aspect per topic. We compared the results to the GPT-LN and GPT-Rank models, using the same measures as described in the main text. Doing an internal manual assessment of a sample of 40 GTs for each model, we found that adding aspect context did not improve the plausibility and relevance of GTs, not even when introducing heuristics to detect aspects that are more relevant to the topic. A possible explanation for this is that the selection of appropriate aspects should be handled more carefully (e.g., as in Schiller et al. (2020)). Such an approach is beyond the scope of this work, and we leave it for future work.

\section{Using Claim Detection to Rank GTs}

When constructing our pipeline, we examined 3 models for ranking GTs according to their coherence and relevance, concluding that the Claim Detection (CD) model is most successful. This model is obtained by fine-tuning BERT on a similar dataset to what was used to fine-tune GPT-LN (the main difference is that the data used to finetune BERT included also negative examples from the same corpus), thus reminiscent of bootstrapping. Indeed, this method of using a classifier finetuned on the same data as GPT- 2 to filter generated samples has already proven to be effective in the context of augmenting low-resource datasets with generated texts (Anaby-Tavor et al., 2019).

\section{Lists of topics}

\section{D.1 List of dev set topics}

We should legalize doping in sport We should protect endangered species

We should legalize insider trading

We should lower the drinking age

We should abolish temporary employment

We should ban free newspapers

We should abolish the US Electoral College

We should ban lotteries

We should legalize ivory trade

We should further exploit green technology

We should ban abortions

We should further exploit geothermal energy

We should raise the retirement age

We should ban alternative medicine

We should subsidize public service broadcasters

We should abolish term limits

We should abandon Gmail

We should not subsidize single parents

We should introduce school vouchers

Prenatal diagnosis should be mandatory

We should prohibit tower blocks

We should increase airport racial profiling in the United States

We should increase international volunteering

We should subsidize the human mission to Mars

The use of AI should be abandoned 


\begin{tabular}{|c|c|c|c|}
\hline Topic & GT & Label & Comment \\
\hline $\begin{array}{l}\text { We should ban alcohol } \\
\text { advertising }\end{array}$ & $\begin{array}{l}\text { alcohol advertising is a form of harass- } \\
\text { ment towards those who may be a heavy } \\
\text { drinker/drinker. it goes against the most } \\
\text { basic human rights. }\end{array}$ & implausible & $\begin{array}{l}\text { The text is coherent, and for many topics saying that } \\
\text { something is a harassment to the point of violating ba- } \\
\text { sic human rights is often a plausible argument. But say- } \\
\text { ing that alcohol advertising is a harassment of drinkers } \\
\text { doesn't make enough sense to be a plausible argument. }\end{array}$ \\
\hline $\begin{array}{l}\text { We should abolish com- } \\
\text { petition laws }\end{array}$ & $\begin{array}{l}\text { competition laws are unfair to those who } \\
\text { don't have the money to hire a lawyer to } \\
\text { get away with murder. }\end{array}$ & implausible & $\begin{array}{l}\text { Here, too, the text is coherent. However, while it's not } \\
\text { clear why competition laws make lawyers more expen- } \\
\text { sive (usually competition drives prices down), the argu- } \\
\text { ment in implausible because getting away with muder is } \\
\text { not something people are likely to argue for. }\end{array}$ \\
\hline $\begin{array}{l}\text { We should disband the } \\
\text { United Nations Security } \\
\text { Council }\end{array}$ & $\begin{array}{l}\text { The United Nations system should be re- } \\
\text { formed to ensure that the council serv- } \\
\text { ing as the ultimate check on the world is } \\
\text { a more efficient mechanism to deal with } \\
\text { global issues. }\end{array}$ & no stance & $\begin{array}{l}\text { Calling for a reform can be taken as an alterntive to dis- } \\
\text { banding, interpreting the claim as contesting the topic. } \\
\text { Alternatively, it can be seen as critical of UNSC, and as } \\
\text { milder policy in the same venue as disbanding. }\end{array}$ \\
\hline $\begin{array}{l}\text { We should increase the } \\
\text { use of telemedicine }\end{array}$ & $\begin{array}{l}\text { telemarketing can be a effective means } \\
\text { of marketing products and conducting re- } \\
\text { search }\end{array}$ & no stance & $\begin{array}{l}\text { The claim is plausible, but it's not relevant to the topic } \\
\text { - it talks about telemarketing instead of telemedicine. } \\
\text { This is probably due to telemarketing appearing in the } \\
\text { training set. }\end{array}$ \\
\hline $\begin{array}{l}\text { We should disband the } \\
\text { United Nations Security } \\
\text { Council }\end{array}$ & $\begin{array}{l}\text { we should not dissolve the united nations } \\
\text { security council because it is an impor- } \\
\text { tant forum to address global issues such } \\
\text { as how to deal with unique situations } \\
\text { involving nuclear war, natural disasters, } \\
\text { and the like. }\end{array}$ & $\begin{array}{l}\text { plausible and } \\
\text { has stance }\end{array}$ & \\
\hline $\begin{array}{l}\text { Flu vaccination should be } \\
\text { mandatory }\end{array}$ & $\begin{array}{l}\text { mandatory vaccination is a good thing. it } \\
\text { keeps kids safe and ensures that those in } \\
\text { the most need of protection are protected. } \\
\text { it can help provide a stronger immune } \\
\text { system to fight disease and protect them } \\
\text { from harmful situations. }\end{array}$ & $\begin{array}{l}\text { plausible and } \\
\text { has stance }\end{array}$ & \\
\hline
\end{tabular}

Table 7: Examples of GTs generated by GPT-Rank on the test set.

We should fight for Palestinian independence

We should further exploit natural gas

We should abandon democracy

We should ban fishing

We should ban gratuities

We should increase government regulation

Community service should be mandatory

We should further exploit solar energy

Tattoos should be banned

We should support a phase-out of lightweight plastic bags

\section{D.2 List of test set topics}

We should end the use of solitary confinement

We should disband the United Nations Security Council

We should end the use of mass surveillance

Child labor should be legalized

We should cancel the pledge of allegiance to the

flag

We should ban multi-level marketing

We should adopt environmental justice

We should ban media conglomerates

We should end the use of traffic enforcement cameras

We should introduce a national identity card

We should subsidize transportation network companies
We should ban burqas

We should ban conversion therapy

We should introduce the alternative vote

Force-feeding should be banned

We should abandon tabloid journalism

We should legalize LGBT adoption

We should abandon Twitter

We should abandon chain stores

We should further exploit mixed-use development

We should subsidize open access journals

We should end child benefits

We should increase the use of telemedicine

We should abandon the sexual revolution

We should adopt polyamory

We should end the use of bailouts

Begging should be banned

We should adopt catholicism

We should abolish credit scores

We should fight environmental degradation

We should increase environmental protection

Flu vaccination should be mandatory

We should close landfills

We should further exploit filibusters

Minority groups should be protected

\section{D.3 Annotation Task Guidelines}

Figures 1-6 present the guidelines for the plausibility, stance and factual vs. opinion annotation 
tasks, as appearing in the Appen crowd-sourcing platform. 
Figure 1: Guidelines for the plausibility annotation task.

\section{Overview}

In the following task you need to determine for each short text, is it a statement that a human will plausibly make. You may consider criteria such as grammar. clarity. "common sense" knowledge, etc.

\section{Examples}

Text: the government funding for genetic testing because it is on such a huge benefit

No - the text is not grammatically clear.

Text: since background checks are only looking for known dangerous criminals

No - this is not a grammatically complete statement - some continuation is missing

Text: making blood donation free will help promote primary care, and improve access to primary care.

No - this text does not make sense; it is not plausible for someone to discuss "making blood donation free".

Text: the high cost of owning motorcycles encourages people to take matters into their own instead

No - this text is not clear.

Text: The elderly should not be encouraged to live long past the normal life expectancy. They are not healthy and expect their longevity fairly and should be given time to bring up children.

No - the second part of the text is not coherent, and the first part is also questionable.

Text: the atmosphere is getting more acidic as carbon dioxide levels in the ocean are increasing this is putting out of the water plants that we need for drinking.

No - even if the first part makes sense, the mention of plants needed for drinking does not, so this should be rejected.

Text: the right to Internet access is a basic human right

Yes - this is a coherent sensible statement.

Text: The United Nations system as currently designed is inadequate and the lack of common-sense rules and regulations present in many international bodies makes it dysfunctional.

Yes - this is a plausible statement - rules and regulations are forcing the United Nations to work in a bad way.

Text: global warming does not harm the ervironment

Yes - although this is probably a false claim, it is plausible that someone will make this statement.

Figure 2: Example of a plausibility annotation.

sedentary lifestyles cause heart disease, diabetes, and obesity among other issues

Is it a statement that a human will plausibly make? (required)

$\mathrm{O}$ Yes

No 
Figure 3: Guidelines for the stance annotation task.

\section{Overview}

In the following task you are presented with a list of statements in the context of debatable topics and are requested to determine for each statement if it supports the topic ("Pro"), contests it ("Con") or does not have a stance towards it ("Neither").

Note: If the statement is not coherent, you should mark "Neither"

Examples

Topic: We should ban the sale of violent video games to minors

Statement: adolescents that play violent video games are most at-risk for violent behavior

Pro - highlighting the negative aspects of violent video games can be used to support the suggested ban.

Statement: kids playing Doom are not at a greater risk for violent behavior

Con - the statement clearly contests the suggested ban.

Statement: the Olympic Games are important for the economy of host nations

Neither - the statement does not discuss the topic

Statement: violence in video games is a major driving force in how successful the game is

Neither - this statement, while discussing violence in video games, does not take a clear stance towards the suggested ban.

Statement: violent video games model physical aggression and they also reward players for being alert to hostile intentions and for using aggressive behavior to solve conflicts

Pro - this statement suggests that violent video games reward aggressive behavior, thus can be used to support the ban.

Statement: placing a tax on violent video games does not impede freedom of speech

Neither - this statement discusses a separate issue, taxing violent video games, instead of the issue of banning them.

Figure 4: Example of a stance annotation.

Topic "We should ban abortions"

abortion is intrinsically wrong

What is the stance of the statement towards the topic? (required)

Pro

Con

Neither 
Figure 5: Guidelines for the factual vs. opinion annotation task.

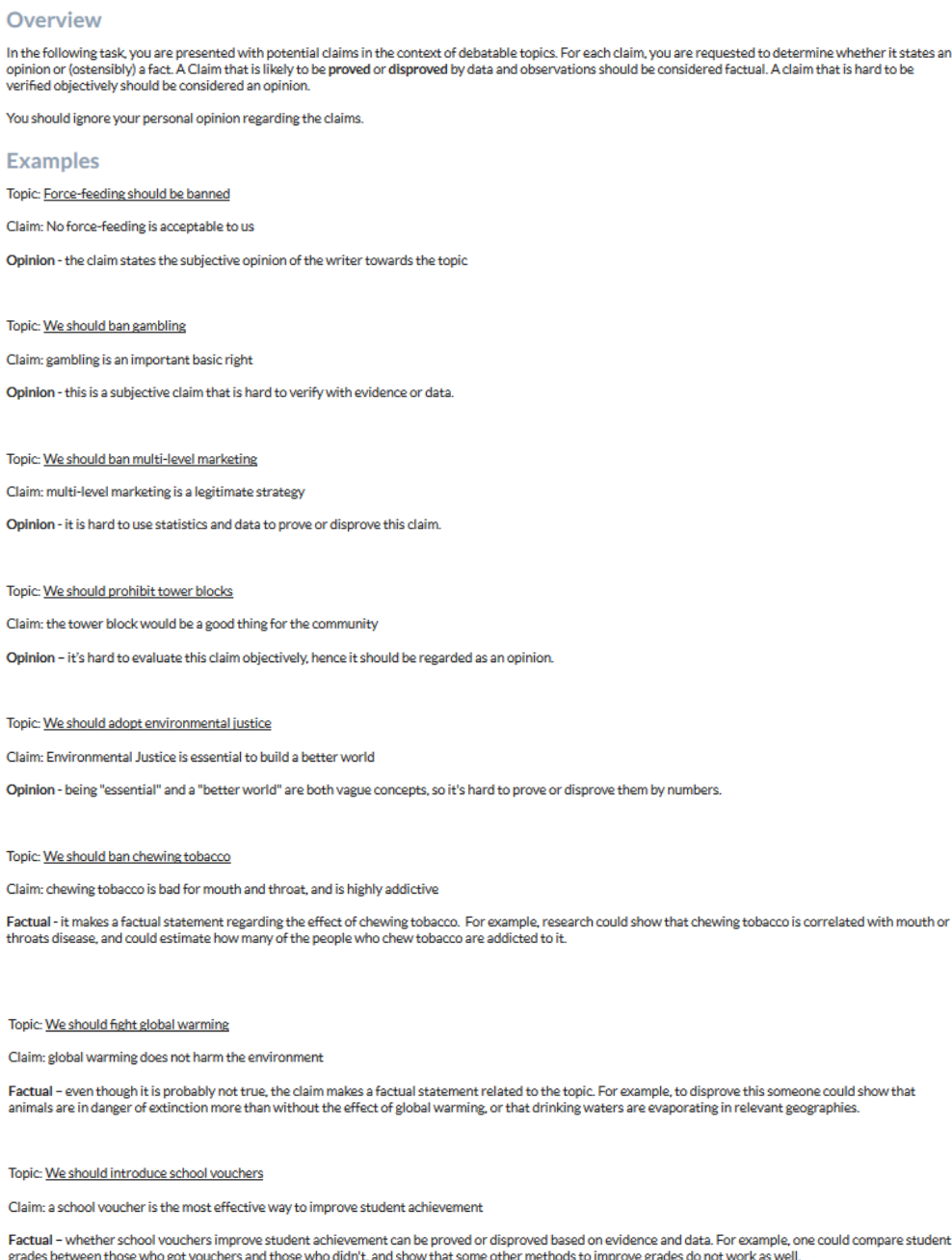

Figure 6: Example of a factual annotation.

\section{Topic "Force-feeding should be banned"}

the force-feeding of pigs is a cruel act

Does the claim state an opinion or (ostensibly) a fact? (required)

Factual

Opinion 